\title{
THE NEW CORONA VACCINE: A WELCOME NEWS BUT WE NEED TO KEEP OUR FINGERS CROSSED
}

\author{
Najib UI Haq ${ }^{\prime \otimes}$, Asif Rehman ${ }^{2}$
}

THIS ARTICLE MAY BE CITED AS: Haq NU, Rehman A. The new corona vaccine: a welcome news but we need to keep our fingers crossed. Khyber Med Univ J 2020; 12(4): 33 I-2. DOI: 10.35845/kmuj.2020.21034.

$\mathrm{V}$ accines are a billion dollars business; World Health Organization (WHO) reports that the vaccine market has grown exponentially in past few years.' It was worth 5 billion in year 2000 enhancing to 24 Billion in 2013 with market growth of $10-15 \%$ per year compared to $5-7 \%$ for the pharmaceuticals and the market volume of vaccines in 2020 is around 60 billion US dollars. ${ }^{2}$

More than 100 companies have been working on corona vaccine across the globe. ${ }^{3}$ The Russian President Vladimir Putin announced in August this year the discovery of corona vaccine and his daughter to be one of trial subjects. Chinese have also developed the vaccine and are conducting phase 3 trials in different countries including Pakistan. ${ }^{5}$ UK and some other countries are working on similar plans, however, none of these could get a response as favorable and the one announced by Pfizer on November $09,2020 .^{6}$

This breakthrough is a sigh of relief for the public and the medical community. Some countries have already reserved orders for millions of doses. The company claims the capacity of preparing 50 million doses by the end of 2020 and another I.3 billion in 2021.6 The cost is not yet clear but estimated to be around 30 US dollars. Irrespective of the medical / political reasons of the widespread positive response to the news, it is important to objectively analyze this welcoming news on scientific evidence and guidelines.

COVID-19 is influenza like virus in its biological behavior but causes much more severe respiratory disease with 5\% patients requiring supplemental oxygen. Unlike DNA viruses (like hepatitis B) it is very difficult to produce effective vaccine against RNA viruses (COVID-19) which do not have fixed regions and have frequent mutations. ${ }^{8} \mathrm{New}$ influenza vaccine is prepared every year against the prevalent mutant/new strains, for improved effectiveness. Mutations have already been reported in COVID virus.'

Efficacy of vaccine: The efficacy of vaccine is judged by its immunogenicity (ability to produce antibodies), safety, stability and duration of immunity. ${ }^{8}$ We still don't know exactly the course of natural immunity of COVID-19 nor sure of possible herd immunity that it may produce. However, it is now reasonably established that prognosis and response to the virus depend on various factors. It is worst in older age and elderly and those with co-morbid conditions including diabetes and cardiac diseases. ${ }^{10}$ This two dose vaccine trial included 43,538 participants and 90 percent of the vaccine candidates have received a second dose of the vaccine till $8^{\text {th }}$ November. ${ }^{6}$ The vaccine is said to be $90 \%$ effective one week after the second dose of vaccine. It's a very short period and we still must wait for the long-term efficacy of the vaccine. The trial conducted on newly discovered vaccine reveals that $42 \%$ of the participants were from diverse ethnic background but it does not explicitly provide evidence of efficacy neither in different ethnic groups nor with respect to age, gender, obesity, severity of illness and co-morbid conditions. The company also declared that at present they have detected 94 cases in the studies population (though did not show the split of vaccine vs placebo group) and that the end point will be detection of 164 cases. That would obviously mean a lower efficacy of the vaccine by the end of trial. The level of vaccine immunity with respect to serial estimation of antibodies (immunity) at various time intervals is also not clear. Response and persistence of immunity with time may be of crucial importance and providing data of quantitative analysis
I. Department of Medicine, Peshawar Medical College, Peshawar, Pakistan.

2. Department of Community Health Sciences, Peshawar Medical College, Peshawar, Pakistan. Email『: professornajib@yahoo.com

Date Submitted: Date Revised: November II, 2020 Date Accepted: December 03, 2020 December 05, 2020

of antibodies of the early inducted individual in trial could have been more informative in determining the continued immunity.

Safety of vaccine: The report of early release of observatory data by Pfizer states that the vaccine is safe. It said "no compromising side effects" were observed but did not provide full information on the type and frequency of side effects observed so far. The Oxford University Vaccine trail was stopped due to side effects (possible spinal cord disease), though the company called it a routine "pause" in the case of an "unexplained illness"." However the United Kingdom experts very rightly called it a good step to hold the trial because the safety of vaccine was above everything else. The trial restarted in October. Provision of data on side effects would have been much reassuring for medical community and the public.

The cold chain: Cold chain is always an important factor in effective vaccination. Changes in optimal temperature levels may grossly affect the efficacy of vaccine and may even render it completely ineffective. The vaccines are traditionally stored around 40 degrees Fahrenheit but this vaccine requires storage temperature of minus 94 degree Fahrenheit. ${ }^{12}$ Maintaining such temperature right from the level of manufacturing to shipping and finally storage, transportation and supply to the end user, may be very cumbersome and cost intensive. This may be an issue even for developed countries requiring millions of doses for mass vaccination. It may be practically impossible for developing countries like Pakistan with hot climate, to fully maintain this protocol of cold chain.

The vaccine may be as safe as projected but the issue of conflict of interest and the "Big Pharma Business" had always been a challenging problem in recent years. 
Ethical issues: Who should be vaccinated first? Should it be the old people at higher risk or the younger with more productivity? There may be ethical issues in priority vaccination of high-risk health care provides ${ }^{13}$, immuno-suppressed groups or those with co-morbidities. These ethical concerns may be in addition to the others vaccine related issue in general.

The enthusiasm: Many drugs were initially used with enthusiasm for treatment of the COVID-19 including chloroquine, remdesivir, steroids, azithromycin and the convalescent plasma. Some of these became nonavailable in market due to bulk purchases by "investors" and even governments. However, the enthusiasm dampened with time because the efficacy/safety could not be proven scientifically in clinical set up. Now is the time and challenge for vaccine. There are still unanswered questions.

It appears that the FDA might give temporary "emergency authorization" for marketing the vaccine as it did in recent months for diagnostics and drugs related to COVID-19. There may be many "pressing reasons" for that. Let us keep our fingers crossed and hope for long term safety and efficacy of corona vaccine. It will be interesting to watch and scrutinize the financial interests/influences of pharma industry versus more transparent and reliable scientific evidence of vaccine safety and efficacy. Let us also hope that the vaccine will be available at affordable prices in future for the benefit of the people across the globe.

\section{REFERENCES}

I. World Health Organization. Global Vaccine Market Report. [Accessed on: November 16, 2020]. Available from URL: https://www.who.int/ immunization/programmes_systems/ procurement $/ \mathrm{mi} 4 \mathrm{a} /$ platform $/$ module 2/MI4A_Global_Vaccine_Market_Re port.pdf

2. Pharmaceutical Processing World. Global Vaccine Market Revenue to Reach $\$ 59.2$ Billion by 2020 Pharmaceutical Processing World. May 2019 [Accessed on: November 16, 2020]. Available from URL: https://www.pharmaceuticalprocessi ngworld.com/global-vaccine-marketrevenue-to-reach-59-2-billion-by2020/.

3. World Health Organization. 172 countries and multiple candidate vaccines engaged in COVID-I 9 vaccine Global Access Facility. [Accessed on: November 16, 2020]. Available from URL: https://www. who.int/news/item/24-08-2020-I 72countries-and-multiple-candidatevaccines-engaged-in-covid-19vaccine-global-access-facility.

4. BBC News. Coronavirus: Putin says vaccine has been approved for use. [Accessed on: November 16, 2020]. Available from URL: https://www.bbc. com/news/world-europe-537357I8.

5. Dawn News. Phase 3 clinical trial for vaccine being developed by China begins in Pakistan. [Accessed on: November 16, 2020]. Available from URL: https://www.dawn.com/news/ I58I02I.

6. Pfizer. Pfizer and BioNTech announce vaccine candidate against COVID-19 achieved success in first interim analysis from phase 3 study. [Accessed on: November 16, 2020]. Available from URL: https://www.pfizer.com/ news/press-release/press-releasedetail/pfizer-and-biontech-announcevaccine-candidate-against.

7. Khorramdelazad $\mathrm{H}$, Kazemi $\mathrm{MH}$, Najafi A, Keykhaee M, Zolfaghari,
Emameh R, et al. Immunopathological similarities between COVID-19 and influenza: Investigating the consequences of Co-infection. Microb Pathog 2020;104554. DOI: 10.1016/j.micpath.2020.104554.

8. Leitner W, Ying $\mathrm{H}$, Restifo N. DNA and RNA-based vaccines: principles, progress and prospects. Vaccine 1999; | 8(9-10):765-77. DOI: I0. 1016/s0264-4I0x(99)0027|-6.

9. Nature. The coronavirus is mutating - does it matter? [Accessed on: November 16, 2020]. Available from URL: https://www.nature. com/articles/d4I 586-020-02544-6

10. Apicella M, Campopiano M, Mantuano M, Mazoni L, Coppelli A, Del Prato S. COVID-19 in people with diabetes: understanding the reasons for worse outcomes. Lancet Diabetes Endocrinol 2020;8(9):782-92. DOI: I0. I0I6/S22I 3-8587(20)30238-2.

II. The Guardian. Oxford University Covid vaccine trial put on hold due to possible adverse reaction in participant. [Accessed on: November 16, 2020]. Available from URL: https://www.the guardian.com/ world/2020/sep/09/oxford-university -astrazeneca-covid-vaccine-trial-puton-hold-due-to-adverse-reaction-inparticipant.

12. Chronicles SF. Pfizer vaccine needs to be stored at minus 94 degrees Fahrenheit. Is the Bay Area prepared to do that? [Accessed on: November $16,2020]$. Available from URL: https:// www.sfchronicle.com/bayarea/ article/Some-coronavirus-vaccinesneed-to-be-stored-at-157| | 275.php.

13. Wise J. Covid-19: Health and care workers will be "highest priority" for vaccination, says JCVI. BMJ 2020;369:m2477. DOI: I0.II36/

\section{CONFLICT OF INTEREST \\ Authors declared no conflict of interest GRANT SUPPORT AND FINANCIAL DISCLOSURE NIL}

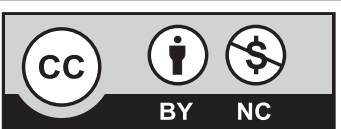

This is an Open Access article distributed under the terms of the Creative Commons Attribution-Non Commercial 2.0 Generic License.

\section{KMUJ web address: www.kmuj.kmu.edu.pk}

Email address: kmuj@kmu.edu.pk 